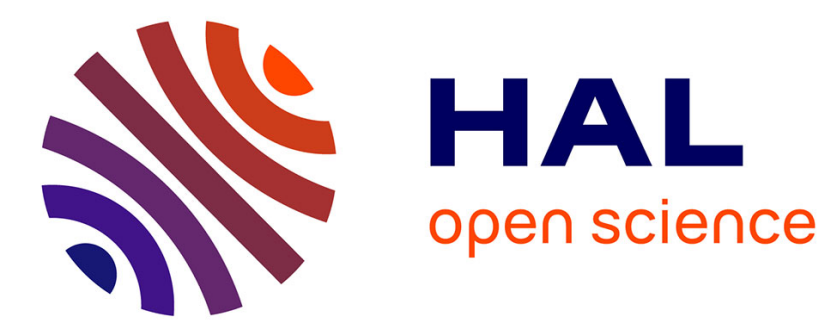

\title{
Phonologies contemporaines : présentation
}

Tobias Scheer, Gabriel Bergounioux

\section{To cite this version:}

Tobias Scheer, Gabriel Bergounioux. Phonologies contemporaines : présentation. Langages, 2015, 198, pp.5-9. 10.3917/lang.198.0005 . halshs-01316866

\section{HAL Id: halshs-01316866 \\ https://shs.hal.science/halshs-01316866}

Submitted on 30 May 2016

HAL is a multi-disciplinary open access archive for the deposit and dissemination of scientific research documents, whether they are published or not. The documents may come from teaching and research institutions in France or abroad, or from public or private research centers.
L'archive ouverte pluridisciplinaire HAL, est destinée au dépôt et à la diffusion de documents scientifiques de niveau recherche, publiés ou non, émanant des établissements d'enseignement et de recherche français ou étrangers, des laboratoires publics ou privés. 
Tobias Scheer

Laboratoire 'Bases, Corpus, Langage' (BCL - CNRS UMR 7320) \& Université de Nice

Sophia Antipolis

Gabriel Bergounioux

Université d'Orléans \& 'Laboratoire Ligérien de Linguistique’ (LLL - CNRS UMR 7270)

\section{Phonologies contemporaines : présentation}

La phonologie est probablement aujourd'hui l'un des domaines les plus inventifs, les plus dynamiques et les plus créatifs des sciences sociales par la nouveauté et la diversité de ses hypothèses et de ses méthodes. Pourtant ses modèles et son axiomatique rencontrent peu d'écho en dehors des discussions que soulève, lors des rencontres spécialisées, la confrontation de différentes théories. De même, sa situation en linguistique est contrastée. D'un côté, elle représente l'un des domaines dont la légitimité est la mieux établie dans les sciences du langage où personne ne songe à contester sa présence. De l'autre, la spécialisation des recherches, par leur technicité ou la spécificité des formalismes, tend à restreindre les interactions internes à la linguistique, et plus encore les transferts vers d'autres champs de recherche, qu'il s'agisse du Traitement Automatique des Langues ou de l'anthropologie.

Il semble, rétrospectivement, que chacun des progrès majeurs accomplis dans l'étude scientifique du langage et des langues ait coïncidé avec (ou plutôt ait été anticipé par) une avancée décisive en matière d'étude du composant sonore des langues, à commencer par l'émergence du comparatisme autour du concept de "correspondance", en passant par la démonstration magistrale de F. de Saussure dans son Mémoire sur le système primitif des voyelles dans les langues indoeuropéennes (1878). À son tour, le corps de doctrine du structuralisme russe s'est fondé sur les propositions de N. Troubetzkoy et de R. Jakobson (1928) avec l'invention de la définition moderne du phonème par le Cercle de Prague, comme le structuralisme américain s'inspirait des analyses de L. Bloomfield (1939) et de E. Sapir (1925). La révision opérée par N. Chomsky et M. Halle avec The Sound Pattern of English (SPE, 1968), comme un élément du dispositif d'ensemble de la grammaire générative, parachèverait cet historique, dans une continuité souterraine avec les écoles qui l'ont précédée. Au-delà de la phonologie, c'est chaque fois l'ensemble de la linguistique qui s'est trouvée reconfigurée.

Dans les dernières décennies, SPE s'est imposé comme le point central de référence. On peut concevoir cet essai comme une synthèse des résultats acquis en Europe - introduits aux États-Unis grâce aux enseignements de R. Jakobson et de 
M. Halle (1956) et de A. Martinet (1945) - et des réflexions concernant la description des langues issues des travaux de L. Bloomfield et de E. Sapir. Pour situer ce projet, on pourrait dire que M. Halle et N. Chomsky se démarquaient à la fois des linguistiques formelles qui n'intégraient pas de volet phonologique (les recherches de Bar-Hillel 1970 ou Harris 1960) et, parallèlement, des connexions qui, en anthropologie, s'étaient établies avec la définition de méthodes de transcription, conçues comme des préalables au recueil de corpus et à la connaissance des cultures.

Alors que les hypothèses en syntaxe - une discipline présentée comme le pivot des sciences du langage -, ont fait l'objet de versions révisées et de rectifications continuelles de la part de N. Chomsky, la phonologie générative s'est trouvée bouleversée sans que cet auteur ne participe véritablement aux discussions qui ont abouti aux reformulations ou à l'abandon de nombre de ses propositions. Le premier exemple concernerait la multilinéarité, introduite à partir du début des années 1970 dans le modèle jusqu'ici uniquement linéaire pour des raisons empiriques et analytiques, sur la base, entre autres, de la tonologie, de la structure syllabique ou encore de l'harmonie vocalique. On en trouve une présentation contextualisée dans P. Encrevé (1988).

Les différentes critiques à l'encontre de $S P E$ et les refontes successives du modèle ont abouti à une multiplication des écoles et des orientations. Il est devenu plus difficile de se repérer et de mesurer les enjeux des nouvelles approches expérimentées en phonologie, une discipline qui s'est considérablement transformée depuis une quarantaine d'années en même temps que la linguistique prenait une dimension nouvelle, tant par le nombre de chercheurs et d'institutions qui y sont impliqués que par le nombre et la variété typologique des langues documentées et analysées.

Sans préjuger de la situation de telle ou telle école, de tel ou tel chercheur, un premier relevé aboutirait à une cartographie paramétrée par trois oppositions majeures :

- la priorité accordée à la description de phénomènes directement observables (inventaire des unités sonores, des constructions syllabiques, des contraintes...)

vs l'élaboration d'écritures symboliques qui représentent, à un niveau abstrait, les séquences et leurs transformations ;

- une détermination empirique des théories à partir de données contrôlées par une accumulation d'occurrences dans des bases de données ou par des expérimentations

vs des conjectures établies à partir de schémas sous-jacents ou qui ont recours à des éléments explicatifs non actualisés en surface ;

- la prise en compte de la variation, qu'elle relève d'une dynamique d'acquisition - ou de perte -, d'un changement dans le temps (diachronique), ou l'espace (dialectologique), ou d'une différenciation sociale (sociolinguistique) 
vs son éviction au profit d'un état de langue considéré comme homogène ou en situation d'équilibre.

En fonction de la part réservée respectivement à la description et à l'explication, à l'empirie et au formalisme, à la représentation et à la dynamique des structures sonores, en tenant compte de leur combinaison, on peut distinguer différents courants en phonologie. Ce numéro de Langages en porte témoignage.

Une autre façon de répertorier la diversité des propositions avancées par les phonologues serait de considérer la façon dont ils sont conduits à formuler des réponses à quelques questions cruciales.

La première concernerait la place de la phonologie dans les sciences du langage. $Y$ constitue-t-elle un domaine disjoint des autres parce que singulière dans son objet ou occupe-t-elle une position bien définie ? Autrement dit, fautil la concevoir comme un niveau de base (c'était l'hypothèse d'E. Benveniste (1966) et, avec certaines nuances, celle de R. Jakobson) ou comme un module interprétatif au sein d'une grammaire centrée autour de la (morpho-)syntaxe qui seule possède le privilège de la créativité, telle que l'a conçue N. Chomsky ? Quelles relations faut-il postuler avec d'autres branches de la linguistique, en particulier avec la phonétique et avec la morphologie ?

Une autre question concerne la valeur universelle des résultats. Nous pensons qu'aucune hypothèse phonologique ne peut être validée si elle ne s'intègre dans un cadre qui ait potentiellement pour capacité de rendre compte, au-delà de ce qu'elle reconstruit, de faits attestés dans des langues de structure très différente, ou plutôt dans toutes les langues avec toutes leurs caractéristiques. Ce travail implique l'usage d'un appareil conceptuel minimal qui assure plus que la compréhension d'un état de langue, celle de ses différentes manifestations, y compris les effets du contact entre les langues et de la cooccurrence des usages.

Les résultats obtenus sont nombreux qui confirment que des progrès significatifs ont été accomplis. Ils ont conduit à réinterpréter l'incidence des catégories articulatoire et acoustique en les hiérarchisant ou en les insérant dans des géométries de traits ou dans des formats associés à la morphologie. Paradoxalement, l'une des conséquences est que la phonologie a fini par devenir un peu moins familière aux chercheurs en sciences sociales et, peut-être même, à nombre de linguistes. Moins faciles à observer et à représenter que les unités segmentales, des concepts comme ceux de contrainte ou de gabarit ne sont pas transposables dans une notation phonétique de type API. À l'inverse, impliquées dans une approche instrumentale et des protocoles d'expérimentation, certaines études de laboratoire peuvent paraître ésotériques à une discipline apparue d'abord aux marges de la philologie. L'écart grandissant entre des formalismes et une analyse concrète du matériau sonore, ou encore l'intérêt renouvelé pour des questions parfois perçues comme secondaires (la liaison, le schwa...) contribuent à une certaine prise de distance avec des analyses qui paraissent difficilement vérifiables ou excentrées. 
Ainsi, la phonologie, en dépit de son prestige et de sa créativité, est peu visible en dehors du cercle des spécialistes. Les mécanismes de financement consacrés par les organismes scientifiques et le jeu des indicateurs conduisent à donner la priorité à des équipements implantés dans des laboratoires ou à l'organisation d'enquêtes à grande échelle. La part « algébrique » (l'adjectif proposé par F. de Saussure) de la phonologie s'y retrouve malaisément. Â l'université, l'enseignement de la discipline, en particulier dans les départements de langues, n'a pas fait l'objet d'une réflexion collective.

En partant de ce constat, il a semblé qu'il était souhaitable d'offrir aux linguistes un panorama de la phonologie d'aujourd'hui. De jeunes chercheurs, travaillant sur différentes langues avec une prédilection pour le français et selon des orientations divergentes, ont bien voulu présenter leur travail comme une illustration des hypothèses qu' ils ont faites leurs. Comme on pouvait $\mathrm{s}^{\prime} \mathrm{y}$ attendre, ils situent souvent leur démarche en référence à $S P E$. La plupart ont déjà eu l'occasion d'exposer une partie de leurs résultats au colloque annuel du Réseau Français de Phonologie. Le fait qu'ils figurent ensemble dans ce numéro est la preuve que la variété des théories n'interdit pas le dialogue et l'échange.

Pour en établir un relevé cursif, les sept contributions s'inscrivent avec :

- Jean-Marc Beltzung dans le cadre de la géométrie des traits qui a contribué, comme Naomi Yamaguchi le démontre, à une révision des propositions en acquisition ;

- Ali Tifrit présente une révision de ce modèle par la phonologie des contours ;

- Julien Eychenne dresse un tableau d'ensemble de la Théorie de l'Optimalité (OT) ;

- Nicola Lampitelli revient sur l'interface phonologie-morphologie en français ;

- Emilie Caratini réexamine le rôle de l'accent tonique en allemand dans un cadre CVCV ;

- Lucille Wallet, enfin, exemplifie l'apport de la Phonologie de Laboratoire (LabPhon) en linguistique clinique.

Avec des exemples empruntés à une dizaine de langues, sur tous les domaines ouverts à la recherche et dans leur affiliation aux principales écoles, ces auteurs livrent un aperçu vivant de l'atelier phonologique contemporain.

\section{Références}

BaR-Hillel Y. (1970), Aspects of Language, Jerusalem / Amsterdam: Magnes Press / NorthHolland Publishing Company.

Benveniste E. (1966), Problèmes de linguistique générale, Paris : Gallimard.

Bloomfield L. (1939), "Menomini morphophonemics ", Travaux du Cercle Linguistique de Prague 8, 105-115.

Chomsky N. \& Halle M. ([1968] 1991), The Sound Pattern of English, Cambridge (MA): The MIT Press. 
EnCREvÉ P. (1988), La Liaison avec et sans enchaînement. Phonologie tridimensionnelle et usages du français, Paris : Éditions du Seuil.

HARRIS Z. (1960), Methods in Structural Linguistics, Chicago: University of Chicago Press.

JAKOBSON R. (1963), Essais de linguistique générale, Paris : Minuit.

JakobSON R. \& HALLE M. (1956), Fundamentals of Language, The Hague: Mouton \& Co.

MARTINET A. (1945), La Prononciation du français contemporain, Paris : Droz.

SAPIR E. (1925), “Sound Patterns in Language”, Language 1 (2), 37-51.

SAUSSURE F. (de) (1878), Mémoire sur le système primitif des voyelles dans les langues indo-européennes, Leipzig : B. G. Teubner.

Troubetzkoy N. \& Jakobson R. (1928), Actes du premier Congrès international des linguistes, Leyde : A. W. Sijthoff. 